\title{
COMPORTAMIENTO POLÍTICO EN LA CIUDAD AUTÓNOMA DE MELILLA EN LAS ELECCIONES GENERALES DE 2011 Y $2015^{1}$
}

\section{Luz Helena Idrobo Bedoya}

Licenciada en Ciencias políticas y de la Administración pública. Máster en Ciencias sociales y jurídicas y en Problemas sociales de la UNED. Doctoranda en Ciencia Política y administración pública. Universidad de Murcia.

Resumen: En esta investigación se pretende determinar cuáles son los aspectos socio-culturales más relevantes de los ciudadanos de Melilla que inciden en su comportamiento político en las elecciones generales. Por tanto, se analizará cómo los ciudadanos de Melilla cambian sus intereses politicos dependiendo de las elecciones y las fuerzas políticas que confluyan en ellas. De esta forma, la investigación se centra en las dos últimas elecciones Generales, proponiendo el estudio de la problemática melillense desde ópticas politológicas y sociológicas a través de la utilización de métodos cuantitativos y cualitativos, centrando el interés en las comunidades más representativas de la ciudad (católicos y musulmanes). Recogiendo con ello, la realidad y caracterización de una población multicultural que coexiste.

Palabras Clave: Comportamiento político, religión, eleccion generales, partidos políticos, análisis del discurso.

Abstract: This research aims to determine which are the most relevant sociocultural aspects of the citizens of Melilla affecting their political behavior in the general elections. Therefore, we will analyze how the citizens of Melilla change their political interests depending on the elections and the political forces that join them. In this way, the research focuses on the two last General elections, proposing the study of the Melilla issue from optical of political science and sociology using quantitative and qualitative methods, focusing the interest in the most representative of the city (Catholics and Muslim) communities. Collecting this reality and characterization of a multicultural population coexisting.

Keywords: Political behavior, religion, general elections, political parties, Speech analysis.

1 Artículo a partir del Trabajo de Fin de Máster-Máster Universitario en Problemas sociales de la Facultad de Ciencias políticas y sociología de la UNED. 


\section{Introducción}

Este artículo recoge la información más relevante de la investigación realizada para el máster Universitario en problemas sociales de la UNED, evidenciando con ello unas pinceladas de su contenido y exponiendo las conclusiones a las que se llegaron. El comportamiento político de los ciudadanos de Melilla varía de una elección a otra y responde a ciertos aspectos sociales, culturales y religiosos. Estas variables son controladas por la multiculturalidad de una ciudad española anclada en la costa del norte de África.

En este sentido la investigación se centrará en dar a conocer y analizar el comportamiento político de los melillenses en las elecciones Generales de los años 2011 y 2015. En este orden de ideas, se tratará de mostrar como unas elecciones fundamentales en la vida democrática española, no lo son tanto para una ciudad con una lógica localista y de partidos políticos basados en ideologías y creencias religiosas.

Por tanto, el objetivo general de este trabajo de fin de máster fue determinar y explicar los rasgos sociales de los ciudadanos de Melilla con el fin de vislumbrar su comportamiento político en las dos últimas elecciones generales. [1]

\section{Principales teorías del Comportamiento político}

La sociedad moderna tiene un papel relevante en la vida política de los Estados, ya que sus ciudadanos son los que desempeñan un rol fundamental en las Democracias. En este sentido, podemos decir, que tienen la opción de elegir, ser elegidos, escoger una iniciativa o adoptar posturas. Estos comportamientos contienen las principales características de la representación política y la teoría de la Democracia.

De aquí, que señalemos en primera instancia, los primeros estudios sobre el comportamiento político llevados a cabo por las escuelas de Chicago y Michigan. En un primer momento, el conocimiento en esta área se centró en el ámbito de la Ciencia política estando su principal instrumento de investigación en los datos y estadísticas, siendo estos el motor del análisis del comportamiento político desde finales de los años cincuenta.

Es de resaltar "la investigación de Lazarsfeld, Berelson y Gaudet, ... The people Choice (1944) la cual establecería un duradero paradigma intelectual al analizar las opciones políticas de los votantes 


\section{TFM}

\section{Ciudadanos de la Ciudad autónoma de Melilla}
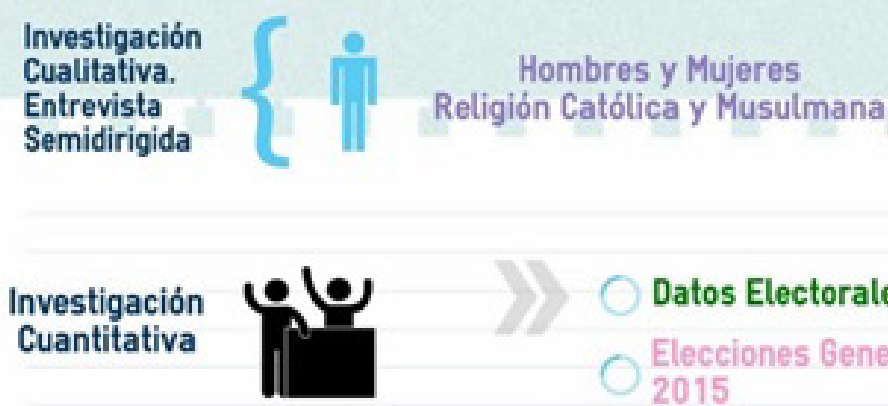

\section{Datos Electorales}

Elecciones Generales 2011 y 2015

\section{Comportamiento Electoral}

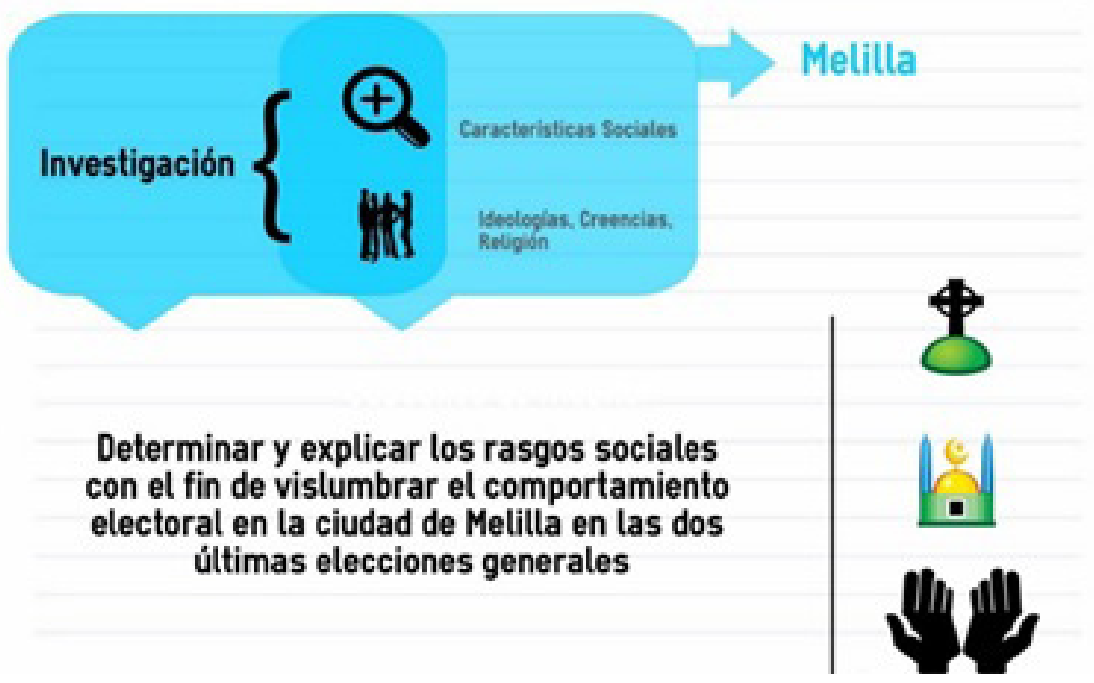

Fig. 1. Contenido del Trabajo de Fin de máster. Elaboración Propia a través de la aplicación easely para infografías en la investigación social.

norteamericanos y tratar de explicar lo observado en forma de datos cuantificables utilizando como herramienta la encuesta" (Delgado y López, 2012, p.157) Esta es reconocida como la escuela de Columbia, la cual entendía que las preferencias políticas son determinadas por las características sociales. Su mayor obstáculo fue la dependencia de encuestas 
con variables reducidas, llegando a ser rebatidas a finales de los cincuenta y comienzo de los años sesenta por las escuelas de Chicago y Michigan.

Asimismo, Lipset un año después revelaría su obra que también sería una novedad para el mundo académico; Political Man, "los principales tópicos discutidos son las condiciones necesarias para la democracia en sociedades y organizaciones: los factores que afectan a la participación de los hombres en la política, particularmente a su conducta como votantes y las bases de apoyo de los valores y movimientos que mantienen o amenazan las instituciones democráticas" Por tanto, según Lipset "Las desigualdades sociodemográficas se traducen en desigualdades políticas”. (Delgado y López, 2012. p.158)

Las investigaciones de Lipset darían lugar a los clivajes ${ }^{2}$. El que nos interesa reseñar para nuestra investigación es el llamado por Lipset y Rokkan como el clivaje Estado-Iglesia el cual se había congelado a partir del endurecimiento de la política de masas en el período de entreguerras. "En ellos se sugería que la religiosidad era un factor decisivo para los votantes a la hora de decidirse por un partido político: no solo la pertenencia a una determinada confesión, sino también la intensidad de las creencias y la práctica religiosa reforzaban las alianzas entre determinados votantes y determinados partidos" (Montero, Calvo y Martínez, 2008. p.22)

A partir de aquí, se inician nuevas investigaciones derivando en un cambio muy importante en el análisis electoral. Este se conocería como el modelo de Michigan. Con su obra "The American voter" Campbell, "expondrían los elementos que explicarían las orientaciones partidistas. Lo cual produciría nuevas formas de pensar en el comportamiento político. Teniendo en cuenta, la identidad político partidaria, imagen de los candidatos, los temas de interés en las campañas, políticas públicas. A partir de aquí se introduce un nuevo concepto que es el de voto por ideología, llegándose a poner etiquetas ideológicas”. (Campbell, Gurin y Miller, citado en Delgado, 2012 p.158)

Igualmente, se hablará en las últimas décadas del "voto reli-

Según el análisis de Stein Rokkan, durante el proceso de modernización de una sociedad se producen una serie de fracturas entre grupos colectivos enfrentados y movilizados en la defensa de sus intereses politicos y económicos. Estas divisiones sociales son susceptibles de cristalizar en partidos politicos, que, en democracia, pasan a definir sus programas y estrategias de acuerdo con el interés representado. Se pueden identificar cuatro (clivajes) escisiones principales que dan lugar a la génesis de la mayor parte de los partidos y son 
gioso", derivado a partir del clivaje Estado-iglesia, "a través del voto religioso, la religiosidad pervive como un factor potencialmente significativo para incidir en el voto. El voto religioso implica una asociación entre la religiosidad y voto que se establece en la intersección entre las estrategias y la evolución del contexto político y social en que se forman las decisiones de los votantes. El acercamiento a la relación entre la religiosidad y el voto desde la óptica del voto religioso, y no del clivaje religioso, la realidad empírica sobre la que se edificó la teoría de los clivajes ha cambiado considerablemente." (Montero, Calvo y Martínez, 2008, p.23)

Los clivajes de Rokkan se han ido reconfigurando a medida que la sociedad se ha transformado. De esta forma, los puntos de ruptura religiosos han cambiado y lo más apropiado sería hablar del auge del voto religioso. El cual implica una necesidad de los partidos por adaptarse a las realidades reales de nuestros tiempos y en especial en el tema religioso debido a la expansión y arraigo de las creencias religiosas, sobre todo en una ciudad como Melilla.

Este desarrollo de ideas, nos permite establecer una relación con el presente trabajo de investigación puesto que se tratará de mostrar si hay una influencia del voto religioso en la ciudad y si ha existido una evolución partidista que congregue la realidad melillense (predominancia de comunidad islámica y cristiana), para de esta forma evidenciar si es posible hablar de la presencia del voto religioso o si por el contrario existe un evolución de los partidos de la ciudad que se muestra en las distintas elecciones.

las que dividen o han dividido a: centro y periferia, con la consecuente emergencia de nacionalismos; campo y ciudad, dando lugar a los movimientos agrarios y librecambistas; religión y Estado, lo que hace surgir los partidos confesionales y laicos; y trabajadores y propietarios.

Es, en todo caso, la tendencia a la moderación y la laicización la que hace que se consolide esta división primordial entre izquierda y derecha. Sólo alli donde continúa politizada la separación entre comunidades etno-territoriales, primordialmente por razones religiosas o lingüisticas, el sistema de partidos y la competición política obedece realmente a otra pauta distinta de la socioeconómica. En este sentido, la aparición de partidos personalizados o vinculados a temas muy concretos no contradice el modelo ya que la idea de Clivaje se vincula a la estructuración permanente y no a fenómenos efímeros. (Molina, 2008, p.21-22) 


\section{Comportamiento electoral de la Ciudad autónoma de Melilla en las dos últimas elecciones Generales (2011-2015)}

En la ciudad de Melilla la vida política es distinta entre los distintos comicios, evidenciándose con ello, la disparidad entre los actores políticos y los votantes según el tipo de elección. En las elecciones nacionales, Melilla escoge un diputado que saldrá del partido con mayor votación y dos senadores bajo la misma fórmula. Al ser una ciudad autónoma este número de representantes es invariable. [2]

\section{ELECCIONES GENERALES ELECCIONES MUNICIPALES}

\begin{tabular}{|l|c|c|c|c|}
\hline \hline & $\mathbf{2 0 1 5}$ & $\mathbf{2 0 1 1}$ & $\mathbf{2 0 1 5}$ & $\mathbf{2 0 1 1}$ \\
\hline Melilla & $53,30 \%$ & $49,43 \%$ & $56,15 \%$ & $58,19 \%$ \\
\hline Nacional & $73,20 \%$ & $68,94 \%$ & $64,93 \%$ & $66,16 \%$ \\
\hline
\end{tabular}

Fig.2. Participación en las elecciones Generales y Municipales de Melilla comparado con los datos nacionales. Elaboración Propia a partir de los resultados publicados por el Ministerio de Interior

Melilla, presenta una baja participación en todas las elecciones, pero en las elecciones generales desde el principio de la Democracia es donde más se ha evidenciado la apatía melillense por los comicios nacionales.

En el dato de la abstención podemos ver claramente que es igualmente alta en la ciudad y comparada con los datos a nivel nacional, se observa que es mayor en las elecciones generales que en las municipales, se ve que en las generales la diferencia ronda los 20 puntos porcentuales entre la ciudad y los resultados nacionales. Mientras que en las municipales son aproximadamente de 10 puntos. [3]

\begin{tabular}{|l|c|c|c|c|}
\hline & \multicolumn{2}{|c|}{ ELECCIONES GENERALES } & \multicolumn{2}{|c|}{ ELECCIONES MUNICIPALES } \\
\hline & $\mathbf{2 0 1 5}$ & $\mathbf{2 0 1 1}$ & $\mathbf{2 0 1 5}$ & $\mathbf{2 0 1 1}$ \\
\hline Melilla & $\mathbf{4 6 , 7 0 \%}$ & $\mathbf{5 0 , 5 7 \%}$ & $\mathbf{4 3 , 8 5 \%}$ & $41,81 \%$ \\
\hline Nacional & $\mathbf{2 6 , 8 0 \%}$ & $31,06 \%$ & $35,07 \%$ & $33,84 \%$ \\
\hline
\end{tabular}

Fig.3. Abstención en las elecciones Generales y Municipales de Melilla comparado con los datos nacionales. Elaboración Propia a partir de los resultados publicados por el Ministerio de Interior

Ahora nos centraremos en describir y analizar los datos cuantitativos en primera estancia sobre las últimas dos elecciones Generales, para luego exponer las opiniones y percepciones de los melillenses frente a la vida política y la forma en que dependiendo de su ideología o confesión 
se comportan políticamente.

Las últimas elecciones generales en la ciudad de Melilla han dejado como resultado la imagen que han señalado los autores citados en este trabajo. Una baja participación electoral y un predominio del Partido Popular, mientras que el Partido Socialista cuenta con poco peso en este tipo de comicios en la ciudad.

La participación en los últimos comicios se incrementó, con respecto de los realizados en el año 2011. El 20 de diciembre de 2015 se registró una participación del 53,30\% mientras que en el 2011 fue de 49,43\%. Este incremento de cuatro puntos nos indica una participación baja con respecto a los porcentajes nacionales. [4]

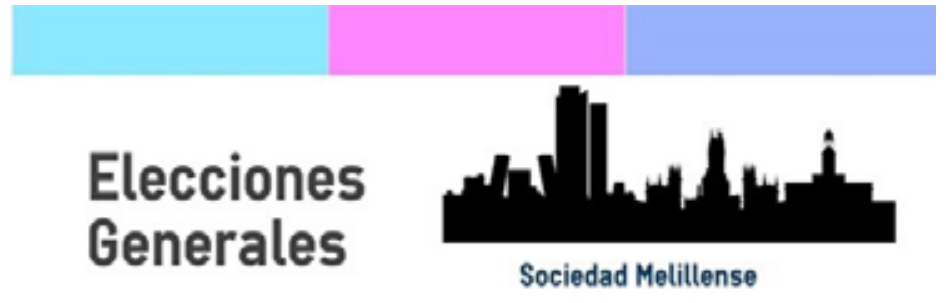

Año 2011 Año 2015

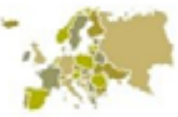

Se presenta un nivel de Participación Menor que en la España Peninsular
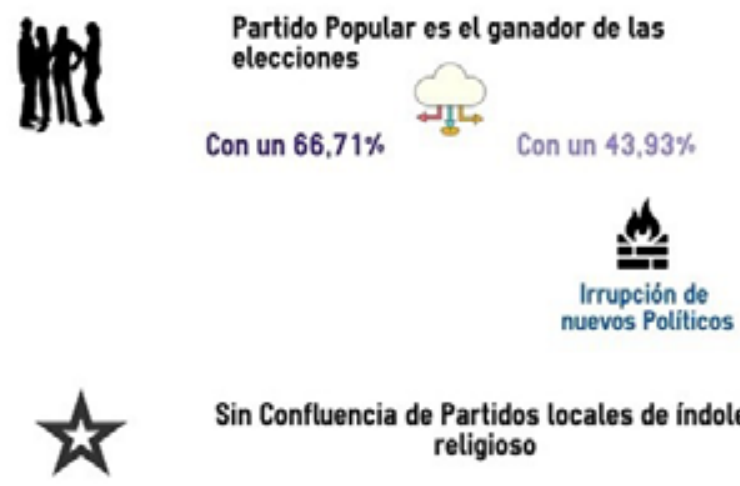

Sin Confluencia de Partidos locales de índole religioso

Fig. 4. Elecciones Generales en Melilla 2011 y 2015. Elaboración Propia a través de la aplicación easelly para infografías en la investigación social 


\section{Análisis cualitativo}

En la investigación cualitativa se encontraron mediante entrevistas semidirigidas cinco discursos principales:

a. Discurso del descontento: Este se encuentra en consonancia con el lado del eje de la participación, puesto que, se da una opinión de descontento a los dirigentes elegidos, además del descontento por las fuerzas políticas que les dirigen a pesar de participar en los comicios.

b. Discurso de la desconfianza: Lo ubicamos en el área de la abstención, siendo propia de los sujetos-pacientes-pasivos quienes perciben que la política es una labor que tiene intereses personales y no involucra al ciudadano. Motivada en el mayor de los casos por las informaciones que reciben de los medios de comunicación sobre corrupción, robo, estafa, etc., mensajes que calan en la opinión y forma de participación, puesto que, incitan a la abstención.

c. Discurso del rechazo: Tiene mucho que ver con el anterior discurso. Pero se reseña en esta investigación que el rechazo al que se hace referencia en los participantes de las entrevistas es a la relación de la religión con la política, todos los entrevistados coincidieron en el rechazo a esta injerencia ideológica en la arena política de la ciudad. Aunque señalaron que es una situación inherente a Melilla debido a la presencia de dos comunidades religiosas mayoritarias (católicos-musulmanes).

A través de la experiencia personal o la socialización con otras personas este discurso se refuerza debido a que se conoce de primera mano que la religión está presente en los debates políticos de la ciudad y configuran una lucha partidista a nivel local.

d. Discurso de la desafección: en este discurso se pone de manifiesto una desafección que también podría llamarse antipatía por la política nacional. También se puede analizar en este punto que existe una desafección por los partidos políticos debido a los escándalos de corrupción y poco interés por los problemas ciudadanos, teniendo así una posición hacia la política pasiva en cuanto a los asuntos políticos y proclives a la abstención electoral.

e. Discurso de la participación: Este discurso fluye hacia la participación en los comicios electorales, a pesar de los sentimientos de frustración, descontento y desafección. La partici- 
pación sobre todo se centra en las opciones políticas de corte local, puesto que, son aquellas que conocen y generan un poco más de cercanía.

Dentro de este discurso también se observa que la participación se realiza hacia una opción concreta según creencias y valores, siendo en la comunidad católica a favor del Partido popular y en la comunidad musulmana más hacia la izquierda o los partidos locales con ideologías islámicas. [5]

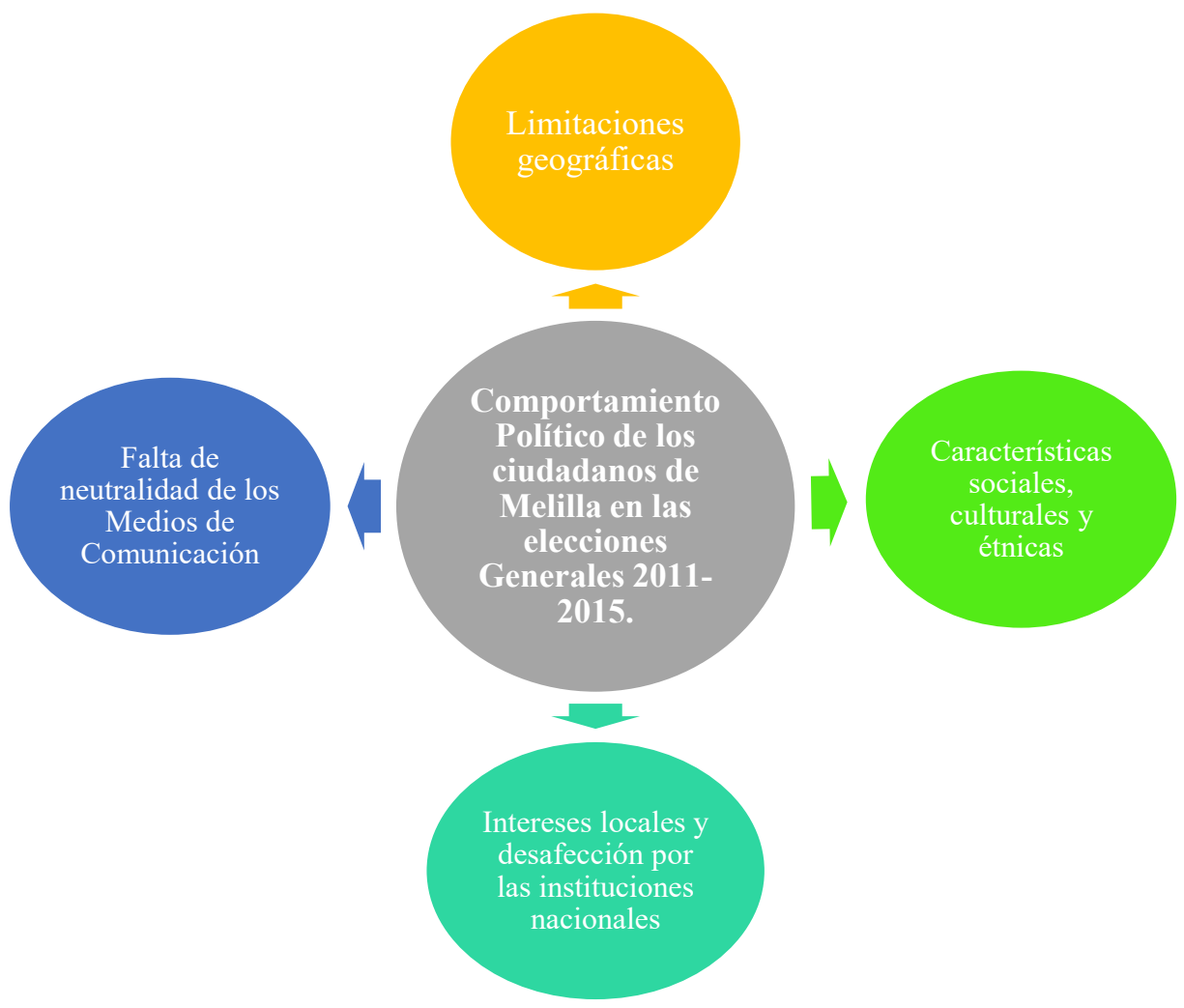

Fig.5. Matriz sobre el Comportamiento político de los ciudadanos de Melilla en las elecciones generales. Elaboración propia, a partir de un análisis de los discursos encontrados en las entrevistas semidirigidas

El comportamiento político de los ciudadanos de Melilla, según los datos extraídos de las entrevistas semidirigidas podría estar resumido en esta matriz, evidenciando lo diferente de la población con el resto 
de España debido a factores geográficos (lejanía y ubicación en otro Continente), sus relaciones sociales (múltiples culturas), desinterés por la política nacional y la falta de neutralidad de los medios de comunicación.

Si relacionamos estos aspectos, nos encontramos con una realidad latente que también se evidenció al exponer los datos electorales, la cual es, la falta de interés en las elecciones Generales que va más allá de la abstención y se materializan en la poca participación electoral en todos los comicios.

Se evidencia como dato positivo, que hay un nivel elevado en conocer todo lo concerniente a los temas locales y todo esto propiciado por la cercanía y el tamaño de la ciudad.

\section{Conclusiones del Trabajo de Fin de Máster}

El comportamiento político ha sido estudiado desde distintas ópticas, nutriéndose de múltiples disciplinas. La ciencia política como pionera de los estudios electorales generó mucho del conocimiento aún vigente en cuanto al comportamiento electoral.

Con la irrupción de nuevos enfoques y sobre todo los aportes de la sociología y la psicología, los estudios del comportamiento electoral dejaron atrás su carácter netamente cuantitativo para ser un conocimiento multidisciplinar que trata de explicar cómo es el comportamiento de los ciudadanos frente a los asuntos políticos teniendo en cuenta sus condiciones culturales, sociales, económicas, religiosas, etc.

En España el comportamiento político de los ciudadanos se empezó a estudiar a partir de la instauración de la Democracia, este momento de la historia española generó unos nuevos escenarios políticos donde las confrontaciones por los ideales del Estado de bienestar se reforzaron y centraron la lucha política.

Hacia los años noventa se entró en una etapa de bipartidismo que enfrentó a las dos grandes fuerzas de derecha e izquierda en el panorama político, la sociedad por su parte, fue cambiando a través de un desarrollo económico acelerado.

Asimismo, se generó un interés por lo que se llama "el voto religioso", en un primer acercamiento, se centra la atención en la iglesia católica, pero con el paso del tiempo, el voto religioso puede analizarse desde ópticas distintas al catolicismo. 
En la ciudad autónoma de Melilla, la entrada de la Democracia también generó ciertos conflictos sociales, sobre todo de tipo étnico y religioso ya que los colectivos que históricamente estaban establecidos en este territorio reivindicaban sus derechos dentro de un país que había cambiado.

El año 1985, fue un antes y un después en la vida política melillense porque fue con la reforma de la ley de extranjería, que Melilla vería incrementada su población con nacionalidad española, generando grandes cambios en el censo electoral y la lucha política.

En las elecciones generales, que ha sido nuestro tema de estudio, el comportamiento observado es distinto al que presenta la población melillense en comparación con las medias nacionales, debido a una desafección por lo nacional, que para muchos parece ser lejano y también gracias a la diferencia en los electorados que pueden concurrir a unas $\mathrm{u}$ otras elecciones.

A las generales solo pueden concurrir votantes de nacionalidad española, mientras que los extranjeros residentes solo pueden ejercer su derecho al voto en las elecciones municipales, situación que condiciona ligeramente la participación en las elecciones nacionales y que provoca también desinterés en algunos ciudadanos de Melilla, debido a la presencia de un porcentaje significativo de personas residentes.

Asimismo, se evidencia la prevalencia de un partido mayoritario desde principios del siglo XXI, el Partido Popular que ha ganado todas las elecciones y que, para las generales cuenta con una amplia ventaja, tal vez debido a la no participación de la segunda fuerza política que es un partido local con gran apoyo de ciudadanos musulmanes que habitan principalmente en la periferia de la ciudad, siendo en muchos casos personas en riesgo de exclusión social por sus condiciones económicas y sociales.

De tal forma, la sociedad melillense presenta unas diferencias marcadas con el resto de España, tanto por su condición de lejanía geográfica con el resto del país, como la confluencia de culturas mayoritarias que se basan en su fe, las dos comunidades más relevantes dentro del conjunto de Melilla (musulmana y católica), están en una coexistencia, pero sus luchas se perciben sobre todo en el ámbito político y que más de la mitad de la población melillense profese el islam genera una lógicas partidistas distintas al resto de la nación. 


\section{Bibliografía}

CORDERO, Guillermo, (2014), "De los clivajes sociales al voto religioso en Europa". Revista de Estudios políticos (nueva época) Número 164. (abril-mayo). Págs. 213-236. Madrid.

DELGADO SOTILLO, I y LÓPEZ NIETO, L. (2012), Comportamiento político y sociología electoral. Madrid. Editorial UNED.

MOLINA, I. (2008), Conceptos fundamentales de Ciencia política. Madrid, Alianza Editorial.

MONTERO, J, CALVO, K y MARTÍNEZ, A. (2008), "El voto religioso en España y Portugal", Revista Internacional de Sociología (RIS), Vol. LXVI, (septiembre-diciembre), págs. 19-54

\section{Referencias electrónicas}

España, Ministerio del Interior. (2014) Resultados electorales desde el año 2000-2011 en Melilla.En: http://www.infoelectoral.interior.es/min/ busquedaAvanzadaAction.html, consultado: 24 de abril de 2016 .

España, Ministerio de Interior. (2016) Resultados al CongresoElecciones 2015. Datos de Melilla. En: https://resultadosgenerales2015. interior.es/congreso/\#/ES201512-CON-ES/ES/CA19, recuperado el 20 de abril de 2016.

España, Ministerio de Interior. (2016) Resultados Elecciones 2015. Datos de Melilla. En:https://resultadosgenerales2015.interior.es/avances/\#/ ES201512-PARES/ES/CA19 consultado el 20 de abril de 2016.

España, Ministerio de Interior. (2016) Resultados Elecciones 2011. Datos de Melilla. En: http://elecciones.mir.es/resultadosgenerales2011/99CG/ DCG19529CI_L1.htm?d=593 consultado el 18 de abril de 2016.

España, Ministerio de Interior. (2016) Resultados Elecciones 2015. Datos nacionales. En: https://resultadosgenerales2015.interior.es/congreso/\#/ES201512-CON-ES/ES consultado el 18 de abril de 2016. 